\title{
Automatic Train Track Switching System with Computerized Control from the Central Monitoring Unit
}

\author{
Md. Reya Shad Azim ${ }^{1}$, Khizir Mahmud ${ }^{2}$ and C. K. Das ${ }^{3}$ \\ ${ }^{1,3}$ Dept. of Electrical \& Electronic Engineering, \\ ${ }^{2}$ Dept. of Electrical Engineering and Automation School \\ ${ }^{1,3}$ Chittagong University of Engineering \& Technology, Bangladesh \\ ${ }^{2}$ Northwestern Polytechnical University, P.R. China \\ azim089@yahoo.com ${ }^{1}$,khizirbd@gmail.com ${ }^{2}$, choton046@yahoo.com ${ }^{3}$
}

\begin{abstract}
A rail track switching system has been designed to control railway track controlling devices including railway switches and signals within a given area from a single point. Such control is exercised through the use of various track circuits which detect the presence of trains on a particular track and monitor the train's safety from a central room. The system secures the safety standards as well as economical and beneficial switch and signal control within a distance maintaining reliability, sensibility and precision. This research is based on microcontroller to reduce the complexity and cost. A low power dc motor is used as a track switching device. In the sensing unit photodiode is used for detecting IR radiation which ensures a reliable detection of trains' entrance. A communication line communicates between the track switching device and main monitoring room. Total system can be monitored and visualize by a software which shows train's position, operation mode and safety status. This system can work both automatically and manually and also can be controlled by the software from the main control room which gives the system more flexibility in operation.
\end{abstract}

Keywords: microcontroller; rail track switching; serial communication; sensor, visual basic

\section{Introduction}

As the major part of the public transport system railway is serving millions of passengers and carrying tones of goods every day. Railways provide a better alternative to other modes of transport by being energy efficient since it can carry large number of people and goods at the same time. As a result, the railways had grown over the years and also the number of people using it. It contributes a lot in our economy. It is their responsibility to have a good management system for good customer services. Therefore, it is necessary for the management to make the train journey safe \& reliable. But the recent train accidents especially head on collisions make the passengers to think otherwise $[1,2]$. The old signalling and operating systems sometimes make wrong operation which causes severe train accident with a huge number of casualties as well as colossal financial losses. In our research, the soul idea was to design a system to avoid the head on collisions of trains due to either disoperation or maliciousness. The system will work automatically and send the information to the central control authorities for further processing. Some sensors have been used to detect the train position and communication line to communicate from the rail track to the main control room. Software will monitor and maintain the whole process to secure the safety of the train. By analyzing cost, efficiency, reliability the system is found better than the existing system. 


\section{System Overview}

The block diagram of the system shown in Figure 1 has four sets of IR transmitter and sensing unit, two of them has been used to detect the entrance or leaving of a train at certain junction (here the track switching point) on one side and the other two sets are used for same on the other side. The two sets of sensor provide data for determining the direction of train course. The others associate block diagrams are depicted in Figures 2 and 3. Based on the signals from the sensors, microcontroller sends the control signal to the track switching motor which switches the rail track to bypass a train in other track to avoid collision. Again there is a provision for manual control of this track from the control room (here, the PC).

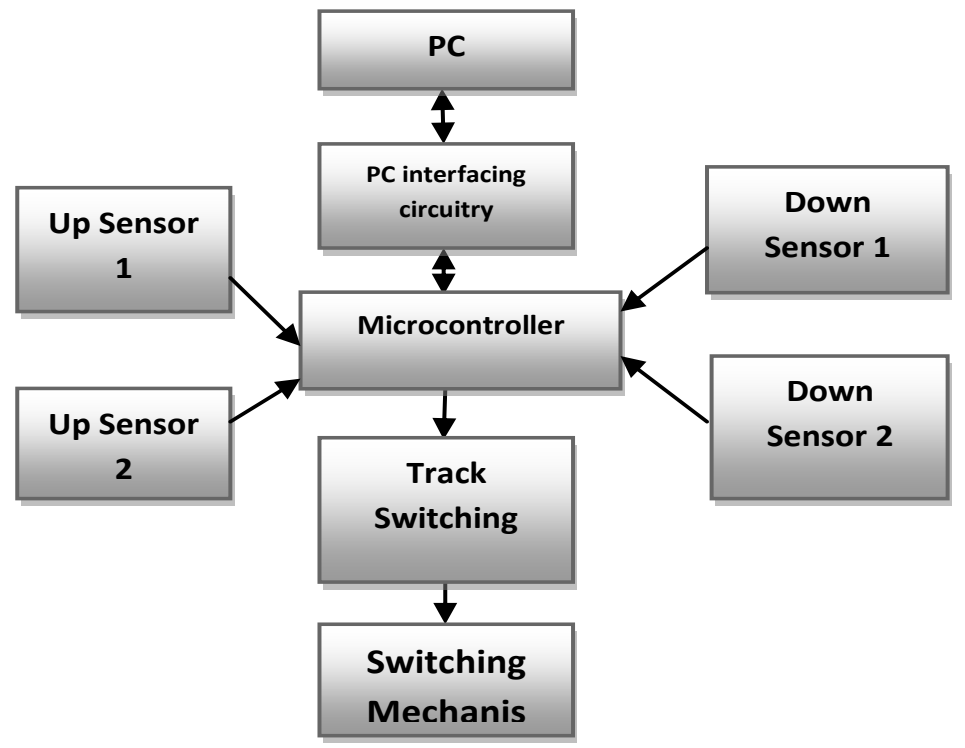

Figure 1. Block Diagram of the System

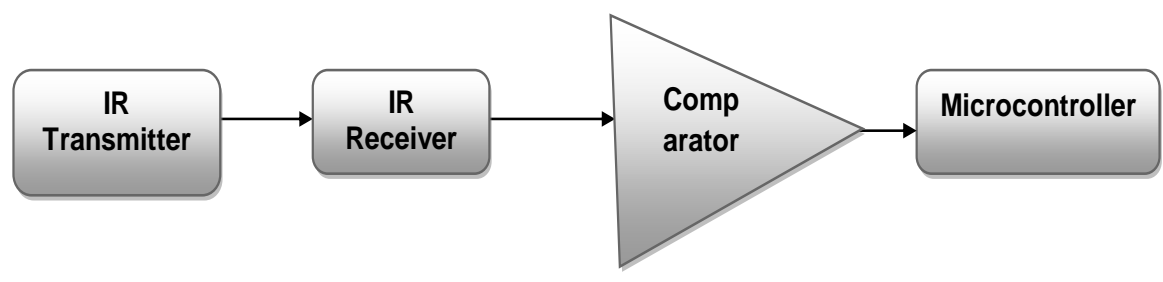

Figure 2. Sensing Unit Block Diagram

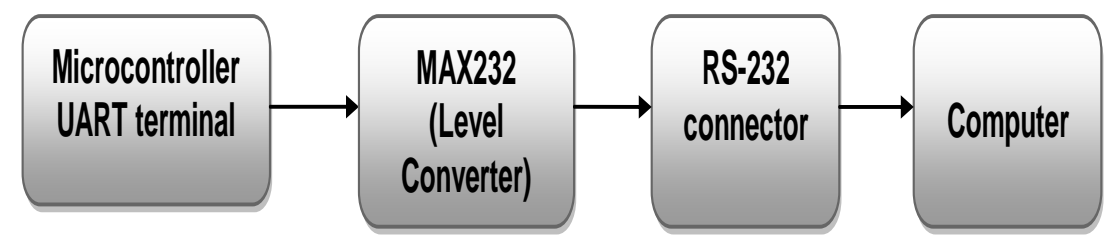

Figure 3. Block Diagram of the Interface between the Main Processing Unit (Microcontroller) and Central Monitoring Unit's PC 


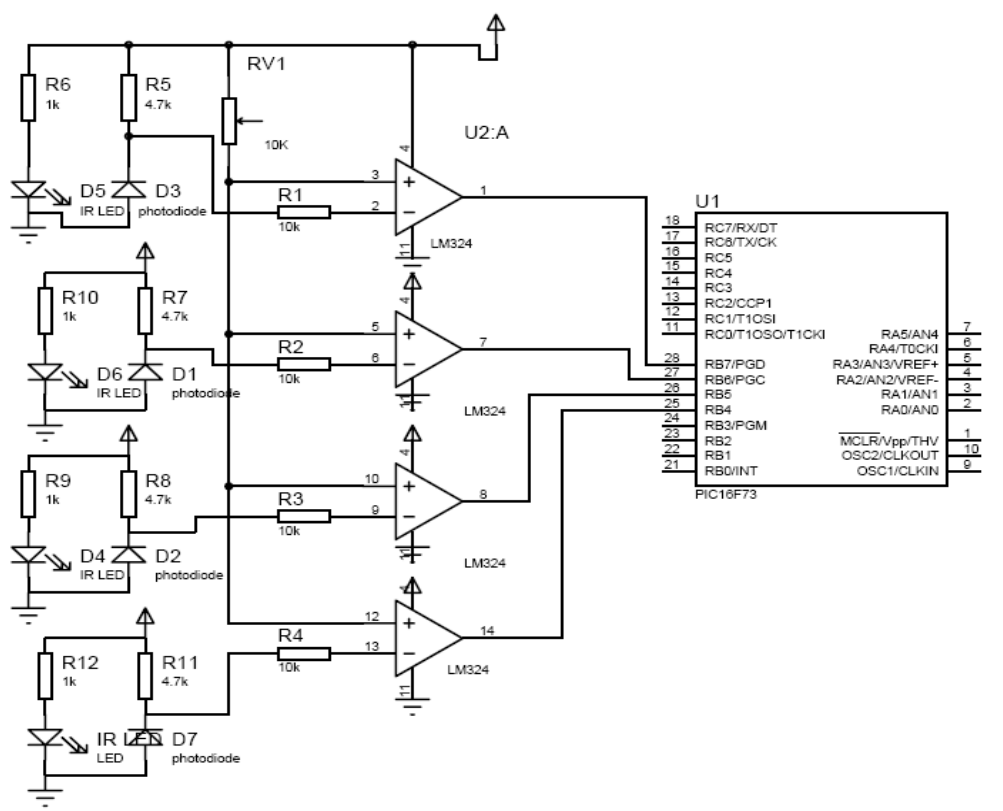

\section{Figure 4. Circuit Diagram for Sensing Units Interfaced with Microcontroller}

Total block diagram integrated with different parts has been shown in Figures 1, 2 and 3. The system is designed for a particular track, if the real implemented system arrangement is complex then the system block and circuit diagram will be different but the main idea will be similar.

\section{System Design}

\subsection{Sensing Unit}

The IR led and the photodiode are the heart of a sensing unit [3, 4]. When no train passes through the sensing unit, then photodiode exposes to IR light which causes photodiode to conducts photocurrent and a low voltage across the diode causing the output of the comparator to be high [5]. When train passes through the sensing unit, then IR light is interrupted and photodiode generates voltage proportional to the intensity of the light and finally made a comparator output $[4,6]$. The detail circuit of all the four sensing units is depicted in figure 4 . The circuit diagram of each sensing unit is depicted in the following Figure 5.

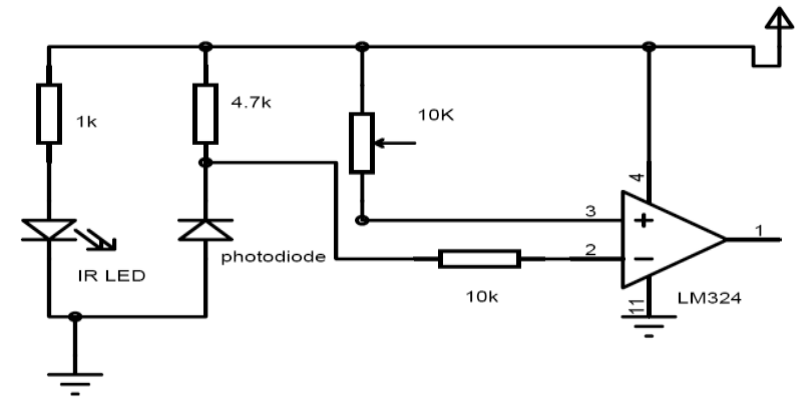

Figure 5. A Single Sensing Unit Set Circuit Diagram 


\subsection{Motor Control Circuit}

The motor control circuit of the system is shown in the following Figure 7.

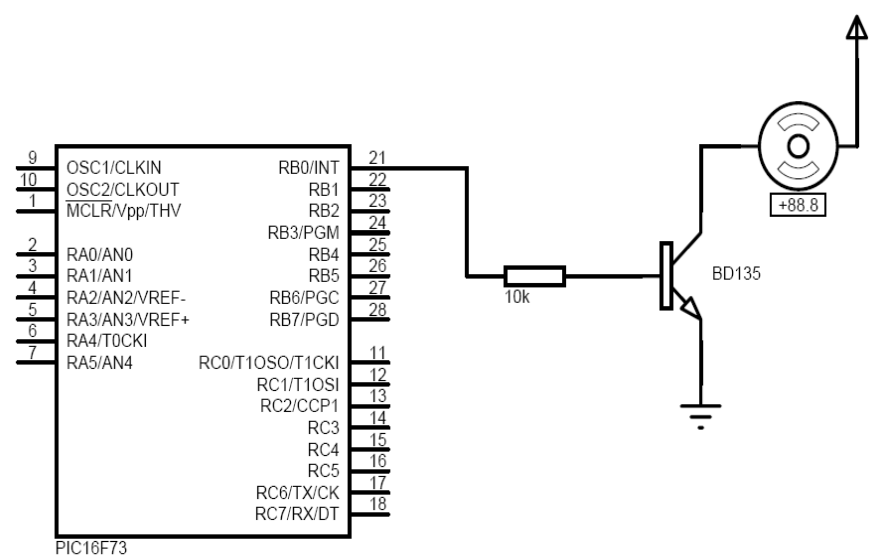

Figure 6. Motor Control Circuit Diagram

When the microcontroller output port is low then the transistor remains off and dc motor operates. When the microcontroller output port is high then the transistor turn on and the motor gets a path to operate as long as the output is high. As the main micro processing unit PIC microcontroller has been used in this system and it can be programmed according to the sensors signal condition [7]. In this microprocessor unit, intelligent fuzzy logic also can be used for better performance and program according to that logic $[8,9]$. The total power system to provide power to the system can be designed as the auxiliary power from the main or renewable solar based or fuel cell hydrogen based also may design [10].

\section{System Working Process}

When a train passes the up sensing unit then the entrance information is stored in microcontroller. If the train is outgoing (out of the junction) the procedure is repeated. If the train is incoming (into the junction) then the down sensing unit status is checked. If the train is outgoing at the down sensing unit, then the operation is again repeated. Otherwise if the train is incoming, while it ensures that two trains are on the way in face-to-face direction, microcontroller sends the information to the pc. If the pc control software is configured as automatic then microcontroller sends signal to the track switching motor to switch track. If the pc control software is configured as manual then microcontroller waits for the controller personnel response and operates accordingly. The overall flow chart of the system is shown in the following Figure 7. A prototype implemented model (shown in figure 8) has two sensing units: Up sensor unit and down sensor unit, also has a track switching unit. Two units separately sense the train's position on a particular track on a particular time. If both sensors sense that the two trains are in a same track then the control software sends signal to the track changing motor to bypass one train from another. Here, track switching should consider about the single track, if the train line is single track then how train will bypass. At that case, have to find the nearest train station where has two line and sensor arrangement \& software algorithm should make in a way so that in a particular distance finding that station the trains bypass each other. 


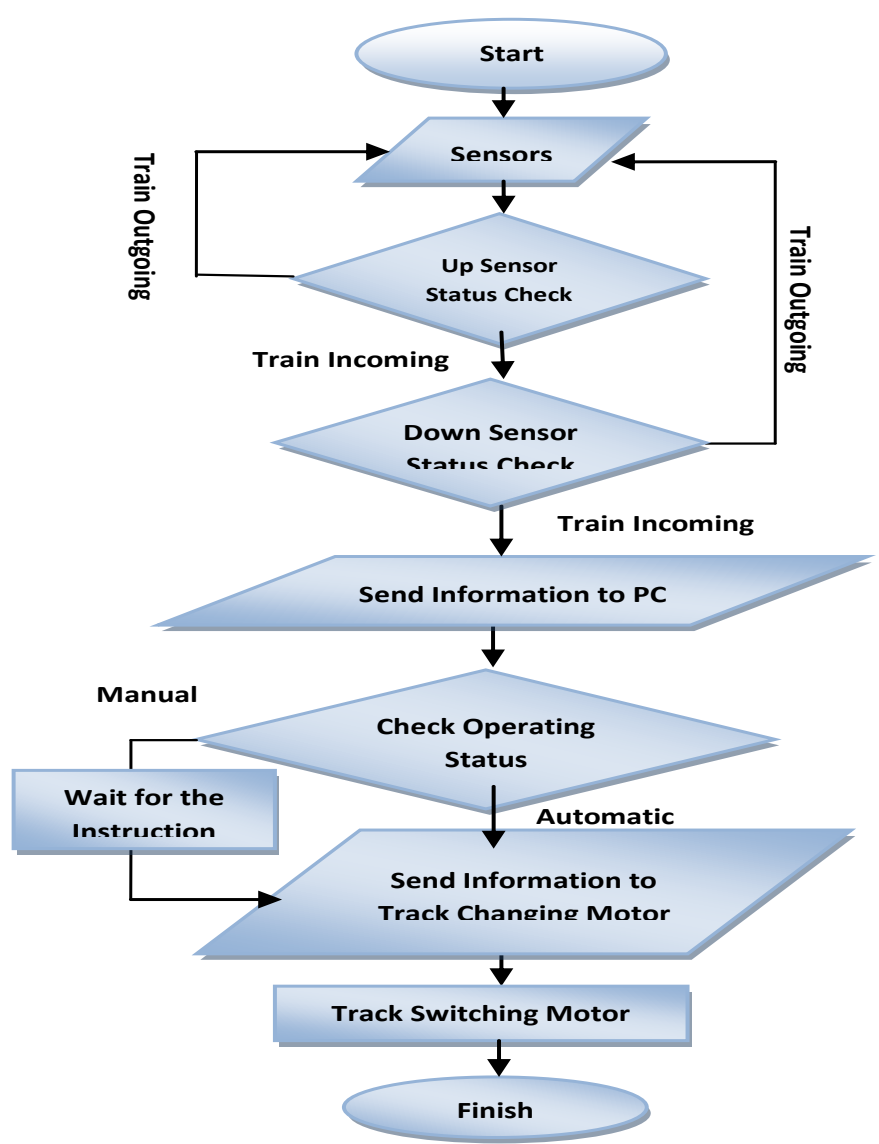

Figure 7. Flow Chart of the Control Software and Sensors Condition in Different Steps

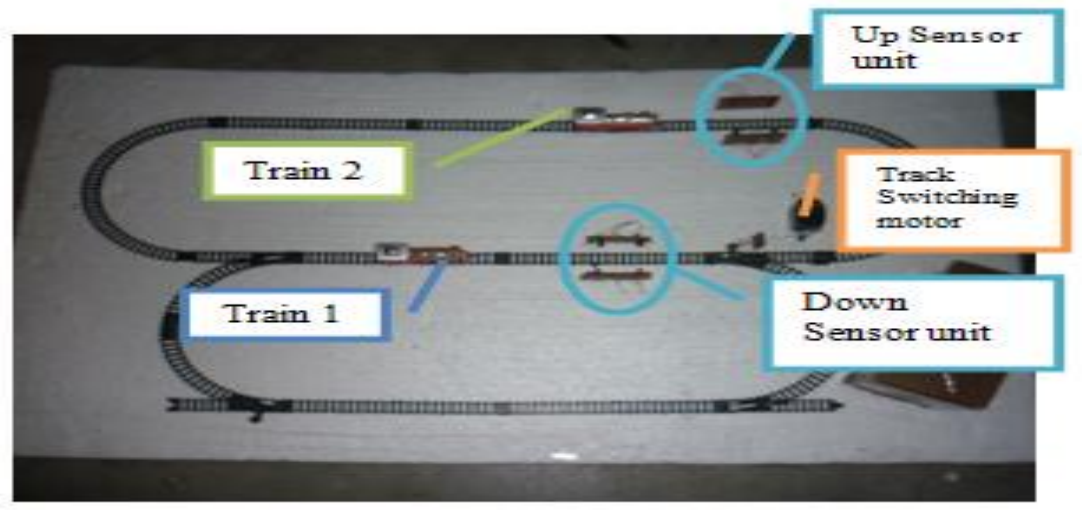

Figure 8. Top View of Entire Implemented System

When the two trains are on each of the tracks, the sensors detects it and send information to the control software. If the control is in 'auto' mode (shown in Figure 9) then the track is automatically switched to bypass one train on the other track. 


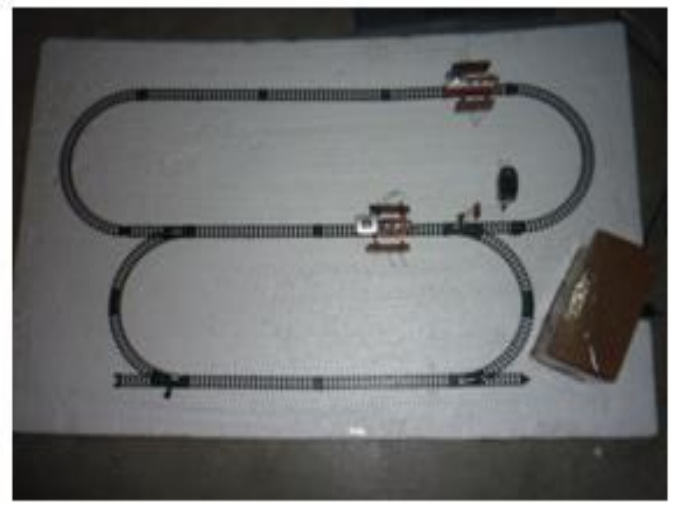

(a) Trains Passing the Sensors Units

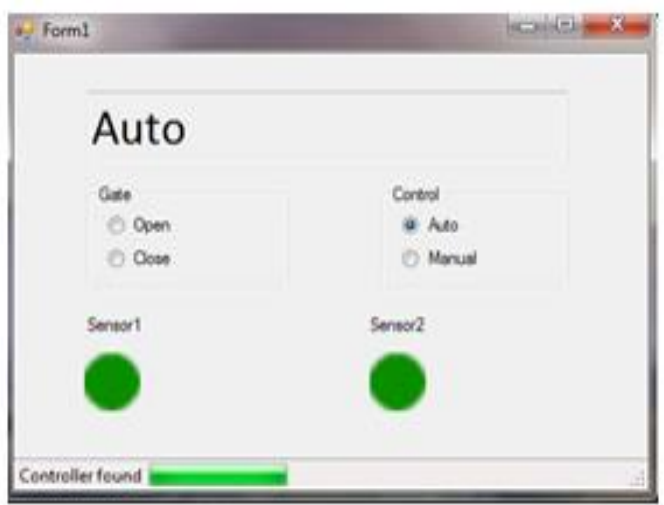

(b) Green Light Indicates Trains' Presence

\section{Figure 9. Automatic Control Depending on the Sensors Information}

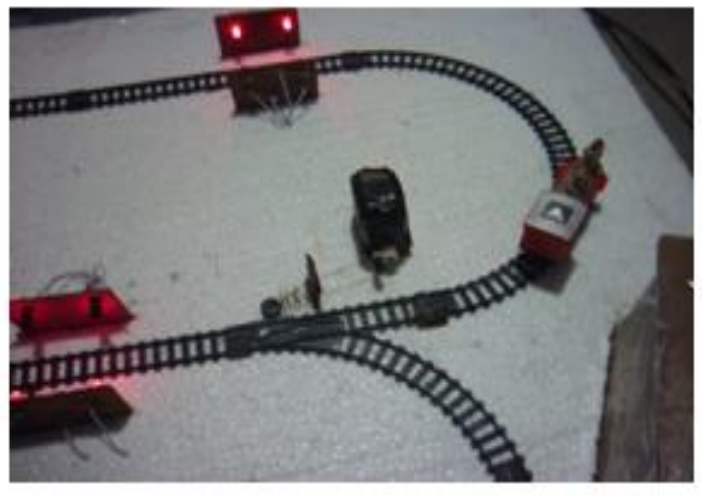

(a) Track \& Train View

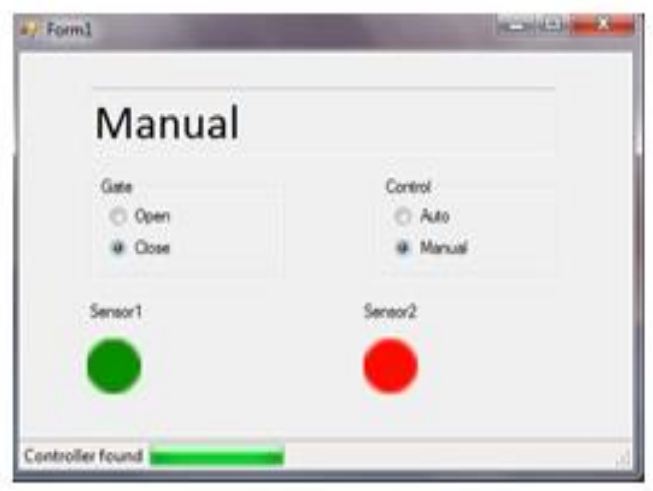

(b) Control Software View

Figure 10. Manual Control Regardless of the Sensor Information (Gate Close)

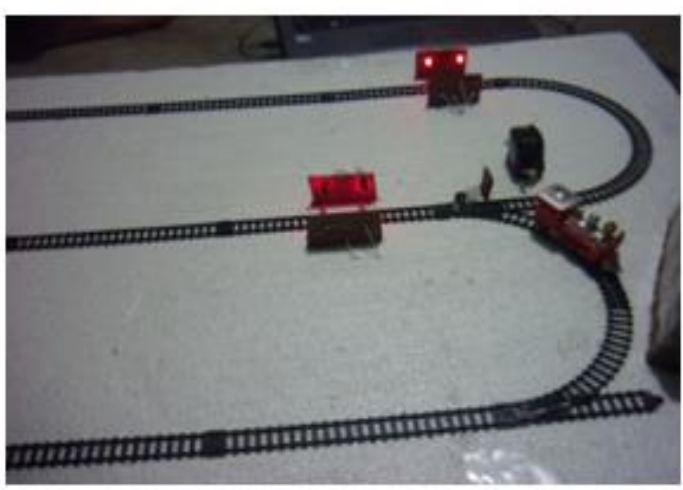

(a) Track \& Train View

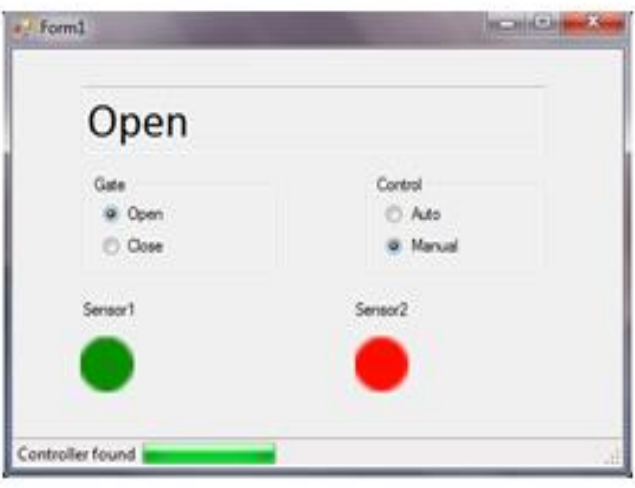

(b) Control Software View

Figure 11. Manual Control (Gate Open) 
In case of 'manual' mode this automatic track switching is restricted and the track switching solely depends on the control software instruction [11]. In this mode when software gate option selected 'close' (shown in Figure 10), the track remains on its normal position. When the gate is in 'open' the track switching motor operates and the track is switched to other position. This condition is shown in Figure 11. In both mode 'auto' \& 'manual' the sensor information is carried to the software window so that the operator has always the indication of the track condition. The control software has another exclusive feature: if any how the control circuit is disconnected from the control PC the software window will indicate it (as shown in Figure 12) and thus the operating authorities can take measures to find the causes of disconnection and will take the necessary steps to recover the system. Though the disconnection from the $\mathrm{PC}$, if the remaining circuits is ok then on emergency condition automatic operation will perform.

\section{Consideration for Practical Implementation}

A prototype of the proposed system has been implemented. For the practical implementation in the railway, some components should change. The sensor parts may consider as requirement of the situation. Here, IR sensor has been used, except this strain gauge or laser or other sensor can be used according to the situation [12, 13]. The main software and other components will function well with all sensors. For implementation, one should consider the single line of the railway and how one train will bypass from other in that line. In this implemented system the software algorithm and sensor arrangements are in such a way so that the train will bypass after coming to the dual track position (as it is known that in the rail line after two/three $\mathrm{km}$ has track crossing system). So according to that condition the software algorithm and sensor position should arrange and by this proposed system's software it can be easily arranged. Another important thing is the link between the sensor and the main control room. In this proposed system, this link is done by the communication cable. In the practical condition, the cable connection may disconnected or other failure can be happen, in this case the proposed software will show indication as 'controller not found' and in that case the system software will work in automatic mode as no monitoring system form the control room. This kind communication failure indication has shown in the Figure 12. But if the distance between the track switching system and the control room is far then fiber optical cable or GSM communication or railway signaling line can be used [14, 15]. This arrangement can be a scope for the further research of the proposed system.

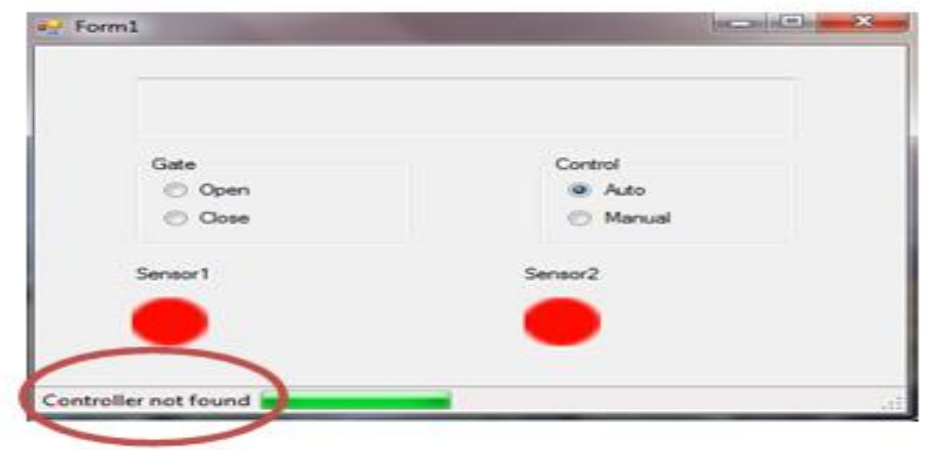

Figure 12. Connection Failure Indication between the Track Switching Circuit and the Monitoring Room and also the Reliable Automatic Controlling Indication in this Worse Case 


\section{Software Design}

For the software design machine language software has been used. The basic software's are micro $\mathrm{c}$ and visual basic. The code has been given below.

$/ /$ microcontroller code

char uart_rd; //Declaring variables

short int one_incoming $=0$;

short int two_incoming $=0$;

short double_mask $=0 \mathrm{~b} 11$;

short one $=6$;

short two $=4$;

short motor $=1<<0$;

short manual $=0$;

short open $=0$;

short curr;

short prev;

void main() //Main program

\{

UART1_Init(9600); // Initialize UART module at 9600 bps

Delay_ms(100); // Wait for UART module to stabilize

trisb=0b11110000; //Declaring portb $4 \mathrm{MSB}$ as input

portb=0; $\quad$ //Declaring portb 4 LSB as output prev=0;

while (1) // Endless loop

\{

if (UART1_Data_Ready()) // If data is received,

\{

uart_rd = UART1_Read(); // read the received data,

if(uart_rd==2)

manual $=1$;

else if(uart_rd==1)

manual $=0$;

else if(uart_rd==3)

open $=1$;

else if(uart_rd==4)

open $=0$;

else if(uart_rd==10)

uart1_write(11);

\}

curr=portb;

if $((($ curr $>>$ one $) \&$ double_mask $)==0$ b10 $) \& \&((($ prev $>>$ one $) \&$ double_mask $)==0)) \quad / / 1->1 \quad 0$ $>0$

$\{$ uart1_write(5);delay_ms(100); one_incoming $=1 ;\}$

if $((($ curr $>>$ two $) \&$ double_mask $)==0$ b01 \& \& $(($ prev $>>$ two $) \&$ double_mask $)==0)) \quad / / 5->04->1$

\{uart1_write(7);delay_ms(100); two_incoming $=1 ;\}$

if(two_incoming \&\& one_incoming)

\{

if(!manual)

$\{$ portb|=motor; one_incoming=0;two_incoming=0;delay_ms(2000);portb\&= motor; $\}$

uart1_write(6);delay_ms(100);uart1_write(8); delay_ms(100); 


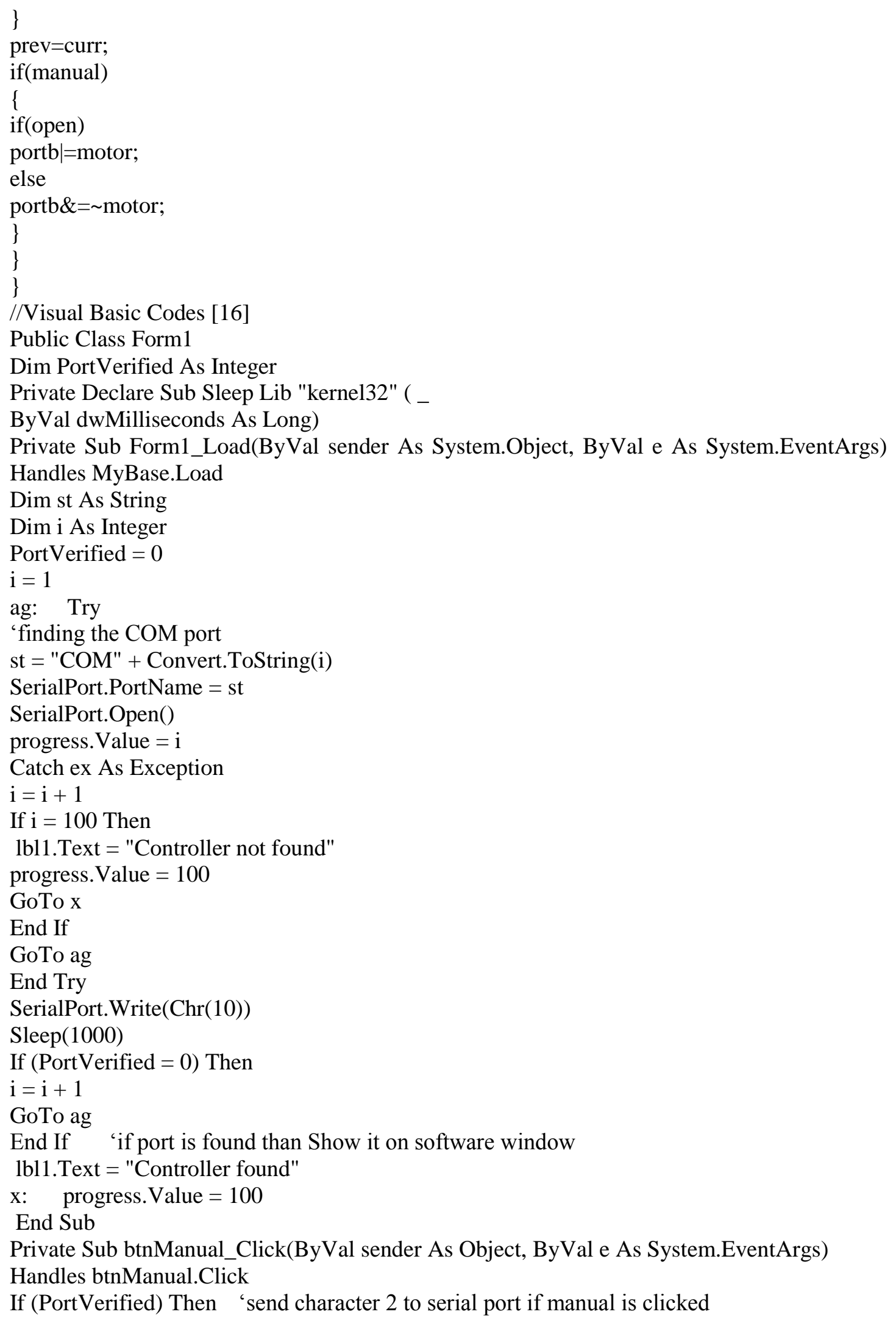




\section{SerialPort.Write(Chr(2))}

txt1.Text = "Manual"

End If

End Sub

Private Sub btnClose_Click(ByVal sender As Object, ByVal e As System.EventArgs)

Handles btnClose.Click

If (PortVerified) Then'send character 4 to serial port if close

is clicked

If (txt1.Text = "Manual" Or txt1.Text = "Open") Then

txt1.Text $=$ "Close"

SerialPort.Write(Chr(4))

End If

End If

End Sub

Private Sub btnOpen_Click(ByVal sender As Object, ByVal e As System.EventArgs)

Handles btnOpen.Click

If (PortVerified) Then

'send character 3 to serial port if open is clicked

If (txt1.Text = "Manual" Or txt1.Text = "Close") Then

txt1.Text = "Open"

SerialPort.Write(Chr(3))

End If

End If

End Sub

Private Sub btnAuto_Click(ByVal sender As Object, ByVal e As System.EventArgs) Handles btnAuto.Click

If (PortVerified) Then

SerialPort.Write(Chr(1))

txt1.Text $=$ "Auto"

End If

End Sub

Private Sub SerialPort_DataReceived(ByVal sender As Object, ByVal e As

System.IO.Ports.SerialDataReceivedEventArgs) Handles SerialPort.DataReceived

Dim inp As Integer

inp $=$ SerialPort.ReadChar()

If (inp = 5) Then

PictureBox1.Image $=$ My.Resources._on

ElseIf (inp $=6$ ) Then

PictureBox1.Image $=$ My. Resources.off

ElseIf (inp $=7$ ) Then

PictureBox2.Image $=$ My.Resources._on

ElseIf (inp = 8) Then

PictureBox2.Image $=$ My.Resources.off

End If

If (inp = 11) Then

PortVerified $=1$

End If

End Sub

End Class 


\section{Conclusion}

The implementation of this automation rail track switching system with computerized control can provide safety to ensure the convenience of railway technology. This research can be used as a safeguard for human life and wealth by avoiding train collision which frequently happens due to wrong operation and mismanagement. It can be implemented easily with less cost and complexity. But some factors like sensor and component selection may be changed according to the environment and situation or desire. According to the specific route or rail track design, some feature of the main controlling software may need to change which can be easily done by this software. In all cases the main features will work similar the implemented prototype. The implementation of the proposed system will contribute a lot in automation of the railway system which will minimize great financial losses as well as the human woe.

\section{References}

[1] M. and K., "Track switching device for two-rail type tracks", U.S. Patent No. 4109584, (1978) August 29.

[2] A. L. Polivka and W. L. Matheson, "Automatic train control system and method", U.S. Patent No. 5828979, (1998) October 27.

[3] A. L. A. T. D. Ambegoda, W. T. S. D. Silva, K. T. Hemachandra, T. N. Samarasinghe and A. T. L. K. Samarasinghe, "Centralized traffic controlling system for SriLanka railways", 4th International Conference on Information and Automation for Sustainability (ICIAFS08), Sri lanka, (2008) December 12-14.

[4] L. H. Peter, "Modern developments in railway signaling", I.E.E. Journal, vol. 78, no. 472, (1936) April, pp. 353-371.

[5] R. F. Coughlin and F. F. Driscoll, "Operational amplifier \& linear integrated circuits", $6^{\text {th }}$ edition, Prentice Hall, USA, (2001), pp. 84-115.

[6] H. S. Petch, "Automatic and semi-automatic railway signaling", IEE journal, vol. 61, (1921) December, pp. 81-88.

[7] K. Mahmud, Md. S. Alam and R. Ghosh, "Design of digital thermometer based on PIC16F77A single chip microcontroller", 3rd International Conference on Consumer Electronics, Communications and Networks (CECNet), IEEE, Xianning, P.R. China, (2013) November 20-22.

[8] K. Mahmud and L. Tao, "Vehicle speed control throught fuzzy logic", Global High Tech Congress on Electronics (GHTCE), IEEE, Shenzhen, P.R. China, (2013) November 18-20.

[9] K. Mahmud, "Neural network based PID control analysis", Global High Tech Congress on Electronics (GHTCE), IEEE, Shenzhen, P.R. China, (2013) November 18-20.

[10] K. Mahmud, "Fuel Cell and Renewable Hydrogen Energy to Meet Household Energy Demand", International Journal of Advanced Science \& Technology, vol. 54, (2013).

[11] F. R. L. Boylestad and L. Nashelsky, "Electronic devices \& circuit theory", $9^{\text {th }}$ edition, Prentice Hall, USA, (2006), pp. 196-199.

[12] A. .H. Cribbens, "Solid-state interlocking (SSI): an integrated electronic signaling system for mainline railways", IEE proceedings, vol. 134, part B, no. 3, (1987) May, pp. 148-158.

[13] G. Dipoppa, G. D' Alessandro, R. Semprini and E. Tronci, "Integrating automatic verification of safety requirements in railway interlocking system design", The 6th IEEE International Symposium on High Assurance Systems Engineering (HASE’01), Washington, USA, (2001) October 22-24.

[14] G. Tarnai, "Safety verification for train traffic control communications", IEEE journal on selected areas in communications, vol. sac-4, no. I, (1986) October, pp. 1118-1120.

[15] J. Axelson, "Serial Port Complete", $2^{\text {nd }}$ Edition, Independent Publisher, (2007), pp. 25-45.

[16] V. Himpe, "Visual Basic for electronic engineering application", $2^{\text {nd }}$ Edition, Elektor-Electronics Publishing, Netherlands, (2002), pp. 204-234. 


\section{Authors}

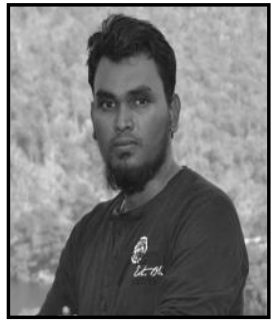

Md. Reya Shad Azim received his B.Sc. degree in Electrical and Electronic Engineering from Chittagong University of Engineering and Technology (CUET), Bangladesh. Currently, he is working as an Engineer in Karnaphuli Fertilizer Company Limited (KAFCO), Bangladesh. His research interests include power electronics and electrical power.

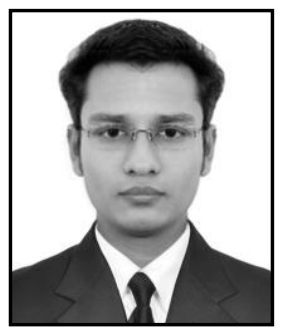

Khizir Mahmud received his Bachelor degree in Electrical and Electronic Engineering from Chittagong University of Engineering \& Technology (CUET), Bangladesh. Currently, he is doing his Masters degree in Electrical Engineering from Northwestern Polytechnical University, P.R. China. His research interests include electrical power, power conversion, power factor, power quality and renewable energy.

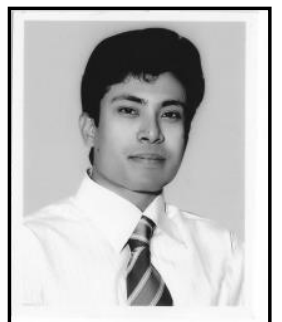

C. K. Das received B.Sc. degree in Electrical and Electronic Engineering from Chittagong University of Engineering and Technology (CUET), Bangladesh. Currently, he is working as an Assistant Professor in the Department of Electrical and Electronic Engineering at Chittagong University of Engineering and Technology, Bangladesh. His research interests include microprocessor, digital electronics and electrical power system. 